Research Article

\title{
Robust Control for Lateral and Longitudinal Channels of Small-Scale Unmanned Helicopters
}

\begin{abstract}
Bao Feng
The School of Electronic Information and Automation, Guilin University of Aerospace Technology, Guilin 541004, China

Correspondence should be addressed to Bao Feng; fengbao1986.love@163.com

Received 26 March 2015; Accepted 16 August 2015

Academic Editor: Zoltan Szabo

Copyright (C) 2015 Bao Feng. This is an open access article distributed under the Creative Commons Attribution License, which permits unrestricted use, distribution, and reproduction in any medium, provided the original work is properly cited.

Lateral and longitudinal channels are two closely related channels whose control stability influences flight performance of smallscale unmanned helicopters directly. This paper presents a robust control approach for lateral and longitudinal channels in the presence of parameter uncertainties and exogenous disturbances. The proposed control approach is performed by two steps. First, by performing system identification in frequency domain, system model of lateral and longitudinal channels can be accurately identified. Then, a robust $H_{\infty}$ state feedback controller is designed to stabilize the helicopter in lateral and longitudinal channels simultaneously under extraneous disturbances situation. The proposed approach takes advantages that it reduces order of the controller by preestimating some parameters (like flapping angles) without sacrificing control accuracy. Numerical results show the reliability and effectiveness of the proposed method.
\end{abstract}

\section{Introduction}

Recently, research on unmanned small-scale helicopters has gained great attention in various fields such as military, industry, and aerial mapping. A striking feature of such unmanned helicopters is the fact that they need to perform different tasks, such as hovering, flying forward, and circular flying, under various complicated situations. Thus, it is necessary to design a robust controller which can stabilize the helicopter in different directions [1-3].

Recently, research on control methods of unmanned helicopters under exogenous disturbances (e.g., wind gust) has received great attention [4-7]. Such kind of classical methods includes PID based control methods [8,9], model predictive control method $[10,11]$, and nonlinear feedforward controller [12-14]. PID control is one of the most classical control approaches whose performance closely depended on the accuracy of the model. In practice, models of control system change with variation of flight conditions. Thus, performance of PID control based methods may be greatly influenced when helicopters perform some difficult movements.
Model predictive control methods are advanced control strategies based on predictions of linear convolution models. Therefore, the model predictive control (MPC) is not a specific control strategy but it is a wide class of optimal control based algorithms that use an explicit process model to predict the behavior of a linear plant. Nonlinear controller can be viewed as ideal, gain-scheduled controller designed at every point on the trajectory. It usually combines with feedback and feedforward technologies to attenuate disturbance of exogenous disturbances.

In this paper, to keep the stability and performance of autonomous helicopter in the presence of parameter uncertainties and exogenous disturbances, a two-step robust control approach based on frequency domain model identification and improved $H_{\infty}$ controller is proposed.

First, we built a simple model for lateral and longitudinal dynamics of a helicopter. Based on the proposed model, a frequency domain method, Comprehensive Identification from Frequency Responses (CIFER) [15, 16], is used to identify the system model. By performing model identification in frequency domain, the proposed method obtained 
robustness against parameter uncertainties and exogenous disturbances.

Based on the identification and modeling of the helicopter, we then designed $H_{\infty}$ state feedback controller to stabilize the helicopter under disturbances situation. A key problem for designing the state feedback control is the fact that the flapping angles are undetectable, which results in the fact that the order of controller is too high to achieve in practice. To overcome this problem, we have estimated the flapping angles by simplifying the speed derivatives in hovering condition beforehand. By this way, order of the controller can be greatly simplified. Thus, the proposed method can efficiently be implemented in practice.

This paper is organized as follows. In Section 2, the lateral and longitudinal channels of a small-scale helicopter are presented and the parameters of the physical structure and model of the helicopter are either identified or estimated in a reliable range. In Section 3, a robust $H_{\infty}$ state feedback control is introduced and designed. In Section 4, the efficiency of the controller designed above is evaluated by analyzing and comparing the results of simulation and real flight. At last, the conclusion lies in Section 5.

\section{Identification of Lateral and Longitudinal Models}

2.1. Lateral and Longitudinal Fuselage Dynamic Model. Considering the hovering flight condition and the Newton-Euler equations [17], the system model for lateral and longitudinal channels can be simplified and linearized as follows:

$$
\begin{aligned}
& \dot{u}=X_{u} u+\left(-w_{0} q+v_{0} r\right)-g\left(\theta+a_{1}\right), \\
& \dot{v}=Y_{v} v+\left(-u_{0} r+w_{0} p\right)+g\left(\phi+b_{1}\right), \\
& \dot{p}=L_{u} u+L_{v} v+L_{b} b_{1}, \\
& \dot{q}=M_{u} u+M_{v} v+M_{a} a_{1},
\end{aligned}
$$

where the constant $g$ is the acceleration of gravity; the vectors $u, v$, and $w$ and $p, q$, and $r$ are the fuselage velocities and angular rates in the body coordinate system, respectively; $\theta$ and $\phi$ are the roll and pitch attitude angles; $X_{u}, Y_{v}, L_{u}, L_{v}, M_{u}$, and $M_{v}$ represent the speed derivatives; the variables $a_{1}$ and $b_{1}$ are the longitudinal and lateral main rotor flapping angles; $L_{b}$ and $M_{a}$ are the flapping spring-derivatives; and $u_{0}, v_{0}$, and $w_{0}$ are the constant values under the trim condition.

Moreover, the main rotor dynamics of such small-scale helicopters can be modeled as

$$
\begin{aligned}
& \tau_{e} \dot{a_{1}}=-a_{1}-\tau_{e} q+A_{\text {lon }} \delta_{\text {lon }}+A_{\text {lat }} \delta_{\text {lat }}, \\
& \tau_{e} \dot{b_{1}}=-b_{1}-\tau_{e} p+B_{\text {lon }} \delta_{\text {lon }}+B_{\text {lat }} \delta_{\text {lat }},
\end{aligned}
$$

where $\tau_{e}$ is the rotor time constant. $\delta_{\text {lon }}$ and $\delta_{\text {lat }}$ are the longitudinal cyclic and lateral cyclic inputs, respectively.

Based on (1) and (5), we can find that lateral and longitudinal channels are coupled, which increases the difficulty of identification $[18,19]$. The main state and control variables for lateral and longitudinal models can be represented by

$$
\begin{aligned}
& \vec{x}=\left[\begin{array}{llllllll}
u & v & \phi & \theta & p & q & a_{1} & b_{1}
\end{array}\right]^{T}, \\
& \vec{u}=\left[\begin{array}{lll}
\delta_{\text {lat }} & \delta_{\text {lon }}
\end{array}\right]^{T} .
\end{aligned}
$$

Under hovering condition, we can get the matrix of dynamic structure in which the procedure is applied to obtain the system parameters. The linearized matrix is illustrated:

$\left[\begin{array}{c}\dot{u} \\ \dot{v} \\ \dot{\phi} \\ \dot{\theta} \\ \dot{p} \\ \dot{q} \\ \tau_{e} \dot{a}_{1} \\ \tau_{e}\end{array}\right]$

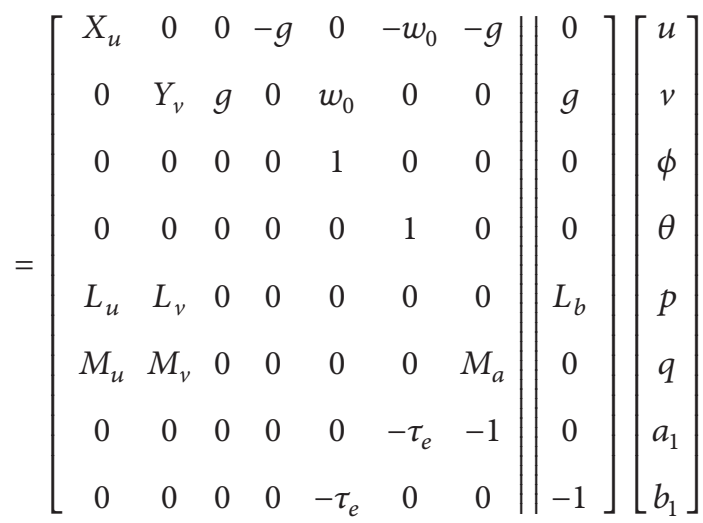

$$
+\left[\begin{array}{cc}
0 & 0 \\
0 & 0 \\
0 & 0 \\
0 & 0 \\
0 & 0 \\
0 & 0 \\
A_{\text {lat }} & A_{\text {lon }} \\
B_{\text {lat }} & B_{\text {lon }}
\end{array}\right]\left[\begin{array}{c}
\delta_{\text {lat }} \\
\delta_{\text {lon }}
\end{array}\right]
$$

2.2. Flight Tests and Collection of Time-History Data. The first step in system identification is the collection of a well-suited time-history database. The final quality of the identification results mainly depends on the executed flight tests of the helicopter. The recommended input for frequency domain identification is a frequency sweep which provides a frequency range of interest for good identification. 

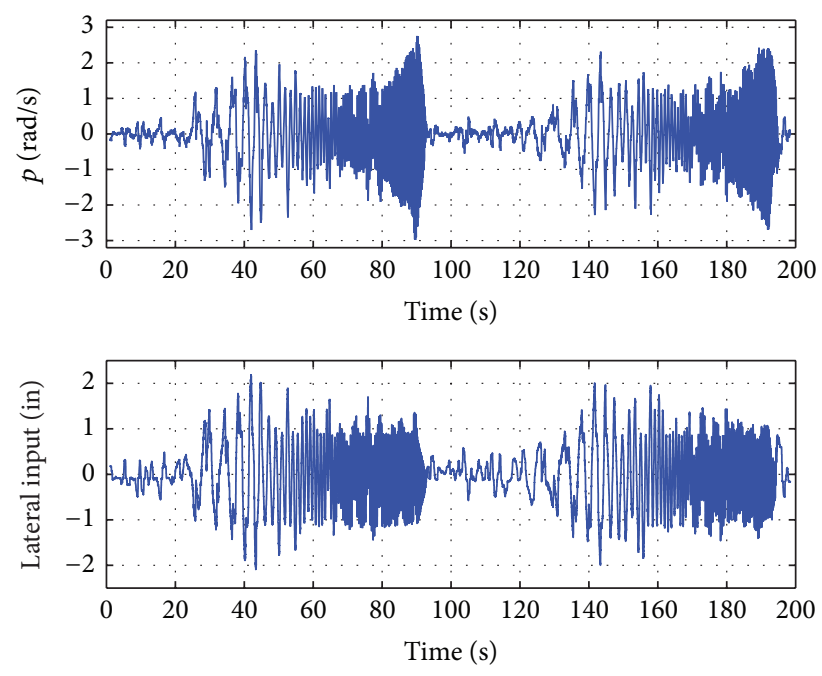

Figure 1: The I/O data of lateral channel.
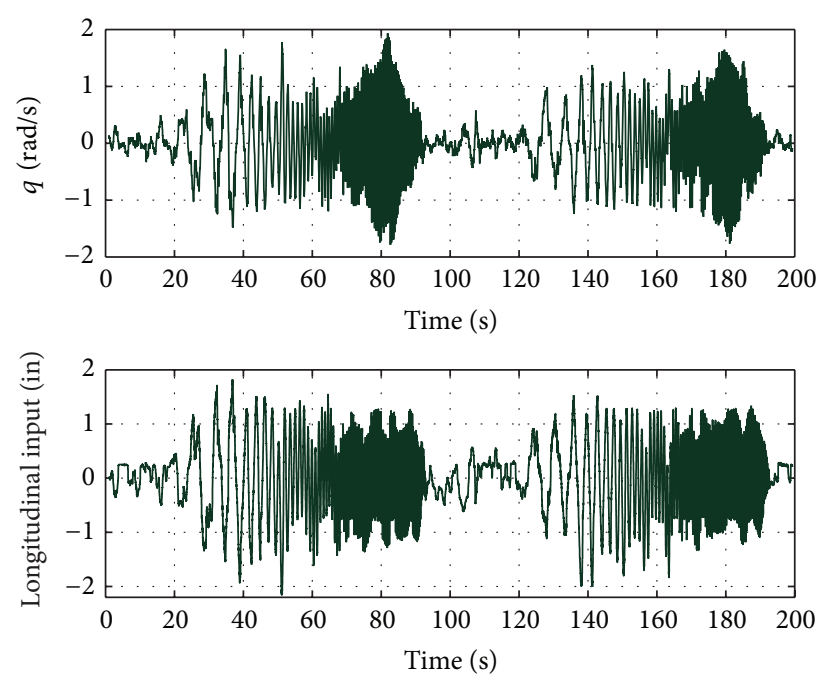

FIgure 2: The I/O data of longitudinal channel.

The maneuver in flight tests being used for identification of frequency responses is a frequency sweep. It is the maneuver that can be performed by either a pilot or automated sweeps. In this paper, all the frequency sweeps are generated automatically, but the pilot still can add his control to the helicopter in case of emergency. The pilot provided a trim record of at least $3 \mathrm{~s}$ before starting the maneuver, and it started with a low frequency sinusoidal shape input with a frequency which would slowly increase. Like [20], the whole process of the maneuver was typically $80-120$ seconds. Figures 1 and 2 show the collection of I/O data of lateral and longitudinal channels, respectively.

2.3. Identification and Estimation of Parameters of Dynamic Model. Around the state of hovering, both the lateral and longitudinal channels transfer functions between the actuator
TABLE 1: The result of identification.

\begin{tabular}{lccc}
\hline Parameter & Value & Parameter & Value \\
\hline$\omega_{n}$ & 27.35 & $X_{u}$ & 0.278 \\
$\zeta$ & 0.565 & $Y_{v}$ & 0.149 \\
$1 / \tau_{e}$ & 9.91 & $L_{u}$ & -0.148 \\
$g$ & 9.77 & $L_{v}$ & -0.237 \\
$M_{a}$ & 426.84 & $M_{u}$ & -0.329 \\
$L_{b}$ & 1338.83 & $M_{v}$ & -0.135 \\
$A_{\text {lat }}$ & 0.179 & $A_{\text {lon }}$ & 6.568 \\
$B_{\text {lat }}$ & 9.487 & $B_{\text {lon }}$ & 0.246 \\
\hline
\end{tabular}

inputs and the angular rates can be approximately presented as

$$
\begin{aligned}
& \frac{q(s)}{\delta_{\text {lon }}^{\prime}(s)}=\frac{\omega_{n}^{2} A_{\text {lon }} M_{a}}{\left(s^{2}+2 \zeta \omega_{n} s+\omega_{n}^{2}\right)\left(s^{2}+s / \tau_{e}+M_{a}\right)}, \\
& \frac{p(s)}{\delta_{\text {lat }}^{\prime}(s)}=\frac{\omega_{n}^{2} B_{\text {lat }} L_{b}}{\left(s^{2}+2 \zeta \omega_{n} s+\omega_{n}^{2}\right)\left(s^{2}+s / \tau_{e}+L_{b}\right)} .
\end{aligned}
$$

Note that the transfer function (9) mainly contains two parts: one for actuator dynamics and the other for helicopter dynamics. Thus, transfer function of the actuator can be given as [21]

$$
G(s)=\frac{\omega_{n}^{2}}{s^{2}+2 \zeta \omega_{n} s+\omega_{n}^{2}} .
$$

Generally, it is difficult to identify the speed damping derivatives $\left(X_{u}\right.$ and $\left.Y_{v}\right)$, because $X_{u}$ and $Y_{v}$ only have significant effect at low frequency when the frequency response precision is usually weak. However, in the actual experiments, we find that these parameters can be estimated as follows:

$$
\begin{aligned}
& \dot{u} \approx X_{u} u-g \theta, \\
& \dot{v} \approx Y_{v} v+g \phi .
\end{aligned}
$$

Hence, by performing a Laplace transform, we can derive the equations and calculate the coefficients $X_{u}$ and $Y_{v}$ :

$$
\begin{aligned}
& \frac{\dot{u}}{q} \approx \frac{-g}{s-X_{u}}, \\
& \frac{\dot{v}}{p} \approx \frac{g}{s-Y_{v}} .
\end{aligned}
$$

The basic parameters in (9), (10), and (13) can be identified by using the transfer function modeling, while others in the state-space model identification can be identified by CIFER. The results are shown in Table 1. 
The state and the input matrices $A$ and $B$ of the linearized model are given as follows:

A

$$
\begin{aligned}
& =\left[\begin{array}{cccccccc}
-0.278 & 0 & 0 & -9.77 & 0 & -0.11 & -9.77 & 0 \\
0 & -0.149 & 9.77 & 0 & 0.11 & 0 & 0 & 9.77 \\
0 & 0 & 0 & 0 & 1 & 0 & 0 & 0 \\
0 & 0 & 0 & 0 & 0 & 1 & 0 & 0 \\
-0.148 & -0.237 & 0 & 0 & 0 & 0 & 0 & 1339 \\
-0.329 & -0.135 & 0 & 0 & 0 & 0 & 426.8 & 0 \\
0 & 0 & 0 & 0 & 0 & -0.1 & -1 & 0 \\
0 & 0 & 0 & 0 & -0.1 & 0 & 0 & -1
\end{array}\right], \\
& B=\left[\begin{array}{cc}
0 & 0 \\
0 & 0 \\
0 & 0 \\
0 & 0 \\
0 & 0 \\
0 & 0 \\
0.179 & 6.568 \\
9.487 & 0.246
\end{array}\right] .
\end{aligned}
$$

For verifying the state-space model, it is conducted with flight data not used in any identification process and the fit ratio indicates the output variation is given as [22]

$$
\text { fit }=\left(1-\frac{\|y-\hat{y}\|_{2}}{\|y-\bar{y}\|_{2}}\right) \times 100 \%,
$$

where the variables $y, \hat{y}$, and $\bar{y}$ represent the real data, predicted data, and the mean of the real data, respectively. The fit ratio indicates how effectively the model predicts the actual output data. Figures 3 and 4 illustrate the comparisons between the actual flight data and the lateral and longitudinal model output data. In Figures 3 and 4, it can be found that both fit ratios are more than $70 \%$, which implies that the model is highly reliable.

\section{Robust $H_{\infty}$ State Feedback Control}

3.1. The Preliminaries of $H_{\infty}$ State Feedback Control. After the system identification and the modeling, the main goal in developing a controller is to ensure that it can stabilize the helicopter in a real environment with uncertainties like uncertain dynamics and extraneous disturbances. It is undeniable that $H_{\infty}$ methodology could not only meet the basic control demands but also minimize the influence of disturbances to the helicopter.

Like the methods mentioned in $[22,23]$, instead of designing a state feedback controller, traditional approach is to use an output feedback controller for the helicopter due to the fact that flapping angles $a_{1}$ and $b_{1}$ cannot be detected. However, the order of such output feedback controller is too high and the structure is complicated, which makes it difficult to use in a real application. To overcome this problem, we use a simple way to estimate the values of the flapping angles,

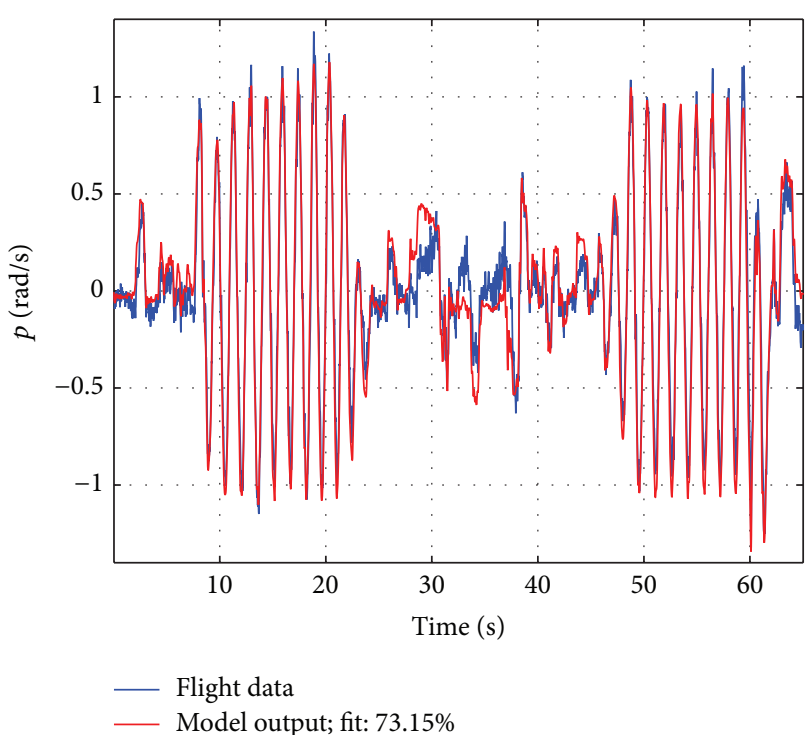

FIgURE 3: The roll rate response of the flight data and the model output.

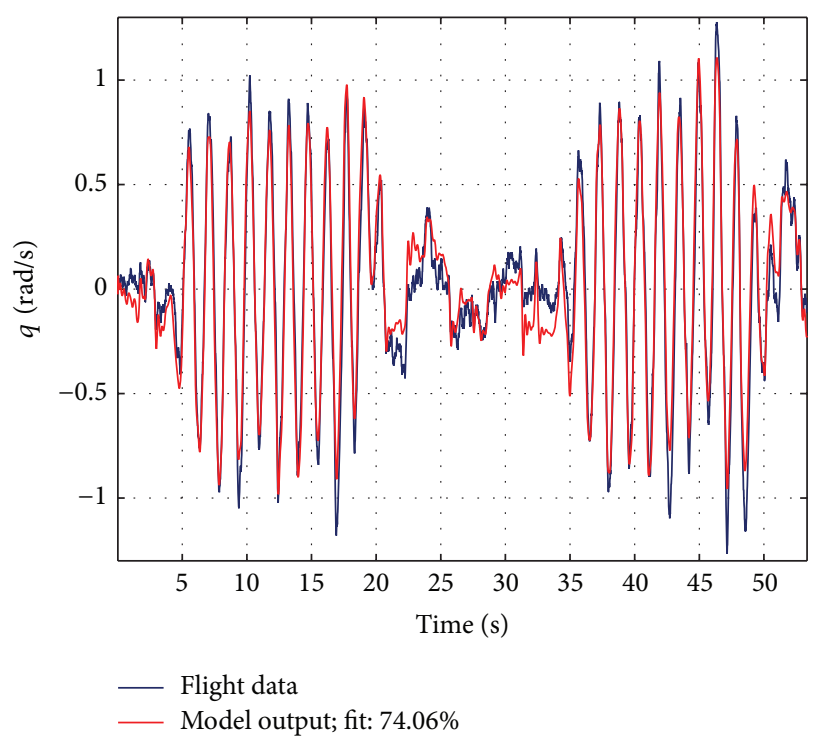

FIGURE 4: The pitch rate response of the flight data and the model output.

neglecting the speed derivatives (in hovering condition); the equations of flapping dynamics can be simplified as

$$
\begin{aligned}
& \dot{q} \approx M_{a} a_{1}, \\
& \dot{p} \approx L_{b} b_{1} .
\end{aligned}
$$

Thus, from (16), we can approximately derive the flapping angle as following transfer function:

$$
\begin{aligned}
& a_{1}(s) \approx \frac{q(s) s}{(\tau s+1) M_{a}}, \\
& b_{1}(s) \approx \frac{p(s) s}{(\tau s+1) L_{b}},
\end{aligned}
$$




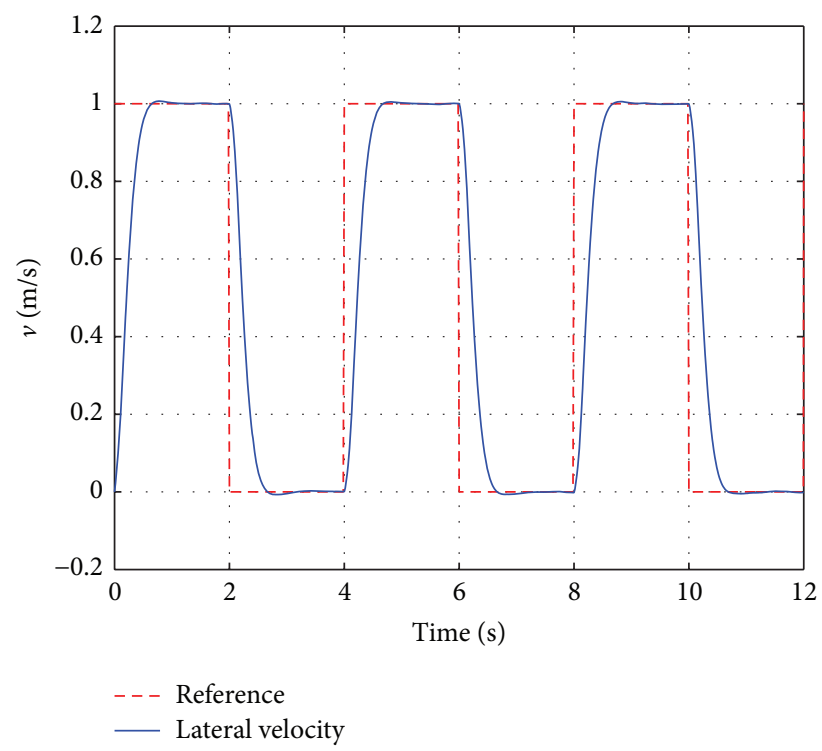

FIGURE 5: The performance of speed tracking in lateral channel.

where $\tau$ is a very small time constant and both angular rates $p$ and $q$ are detectable. With these equations, it is feasible to design $H_{\infty}$ state feedback controller for the lateral and longitudinal channels.

3.2. The Algorithm of $H_{\infty}$ Controller. The dynamic system can be described in a state-space form as follows:

$$
\begin{aligned}
& \dot{x}=A x+B_{1} w+B_{2} u, \\
& z=C_{1} x+D_{11} w+D_{12} u, \\
& y=C_{2} x+D_{21} w+D_{22} u .
\end{aligned}
$$

$$
F=\left[\begin{array}{cccc}
-0.0146 & 0.9634 & 2.3525 & -0.0488 \\
0.9178 & 0.0144 & 0.0322 & 2.8155
\end{array}|| \begin{array}{cccc}
0.1069 & -0.0039 & -0.1136 & 5.8940 \\
-0.0146 & 0.0007 & 5.3074 & -0.0263
\end{array}\right]
$$

respectively. In Figure 7, the changes in roll rate and roll angle last less than $1 \mathrm{~s}$ in a single control period. The results in Figure 8 show that the proposed method can also control the changes of pitch rate and pitch angles less than $1 \mathrm{~s}$. Thus, the proposed controller can stabilize the attitudes of the helicopter in less than $1 \mathrm{~s}$ which demonstrates effectiveness and stability of the controller.

4.2. Performance Evaluation Using Flight Tests. After showing the performance of the controller by analyzing the simulation results, it is necessary for us to evaluate performance of the proposed method in real flight tests under the condition of hovering. Figures 9 and 10 show the actual output (including velocities and attitudes) of lateral and longitudinal channels. In Figures 9 and 10, we can find that the pitch angles eover, the performance of angles and angle rates on and pitch channels can also be seen in Figures 7 and 8,
If the system satisfies the condition,

where variable $X$ is a positive solution of the following algebraic Riccati equations [24]:

$$
A^{T} X+X A+\gamma^{-2} X B_{2} B_{2}^{T} X+C_{1}^{1} C+\varepsilon I=0 .
$$

$\varepsilon$ is a sufficiently small constant. And the controller can be given as

Using the algorithm mentioned above, we select $\gamma_{0}=$ 21.74 , and we can calculate matrix $F$ which is given as follows: close-loop transfer function from $w$ to $z$ can be internally stabilizing controller to ensure the infinity norm iteration [22]. A state feedback $H_{\infty}$ controller which meets the requirement $\left\|T_{z w}\right\|_{\infty}<\gamma$ is given as

$$
F=-B_{2}^{T} X
$$

$$
\begin{aligned}
D_{11} & =D_{22}=0, \\
D_{12}^{*}\left[\begin{array}{ll}
C_{1} & D_{12}
\end{array}\right] & =\left[\begin{array}{ll}
0 & I
\end{array}\right] .
\end{aligned}
$$

4.1. Performance Evaluation Using Simulated Data. In this section, numerical experiments are carried out to evaluate the real data sets.

In order to observe how well the controller can perform for speed tracking, a square wave is used as a tracking command. Figures 5 and 6 show the simulation results channels. In Figures 5 and 6, the proposed method costs less than $1 \mathrm{~s}$ to track the speed parameter with a very small overshoot, which means that the responses of the speed tracking based on the proposed method are fast and accurate. constant $\gamma$. It is called the standard $H_{\infty}$ control problem, 


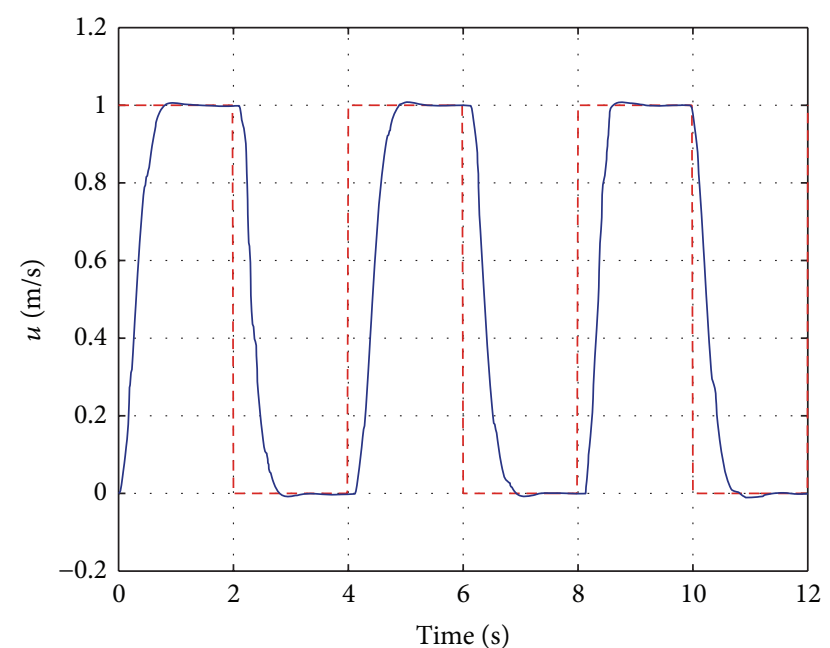

- - Reference

_ Longitudinal velocity

FIGURE 6: The performance of speed tracking in longitudinal channel.

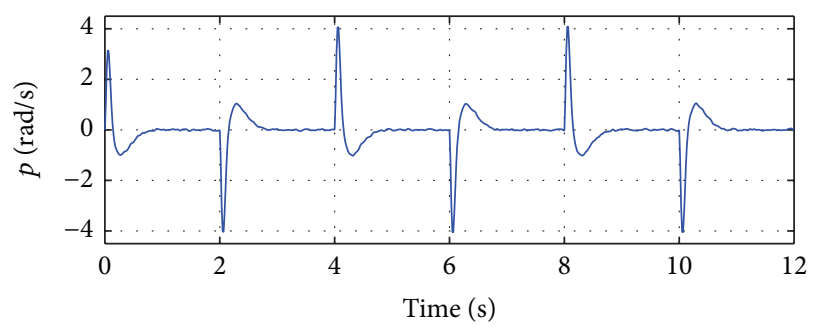

_ Roll rate

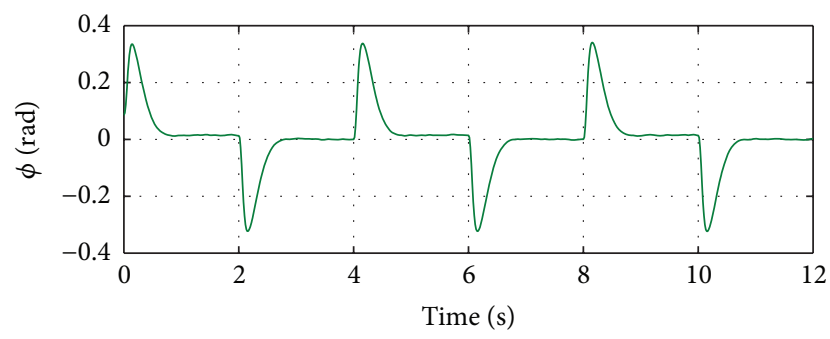

— Roll angle

FIgURE 7: The performance of roll rate and roll angle.

change little, while the roll angels change a little more, which implies that the proposed controller can stabilize the helicopter in a real application effectively. Moreover, after careful observation, we found that the roll angle is not equivalent to zero in Figure 9. This is mainly due to the fact that, under the hovering condition, the main rotor needs to tilt with a small angle to offset the force coming from tail rotor.
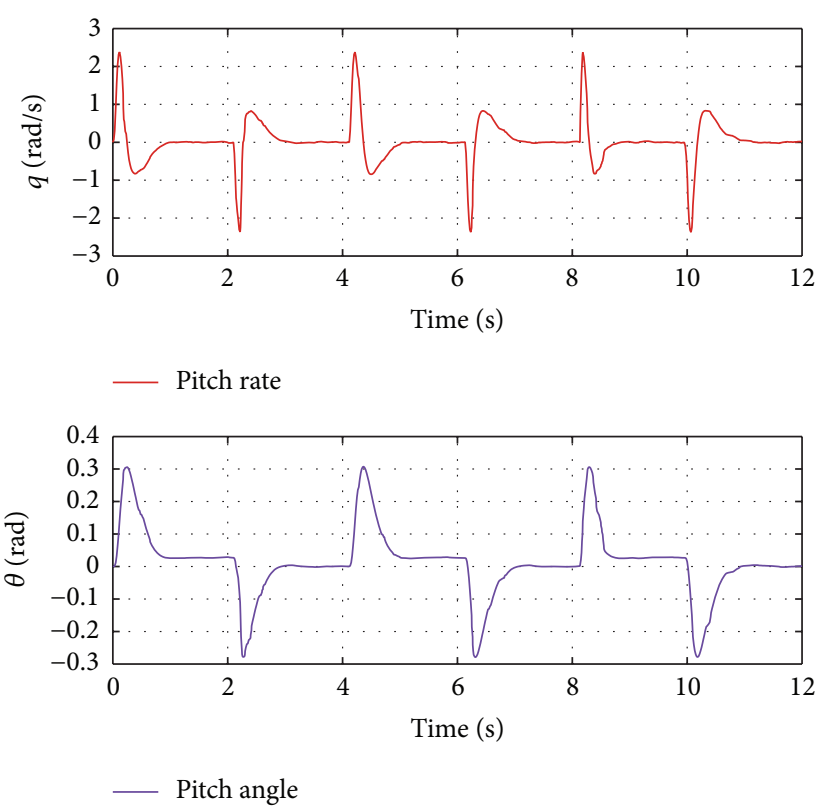

FIGURE 8: The performance of pitch rate and pitch angle.
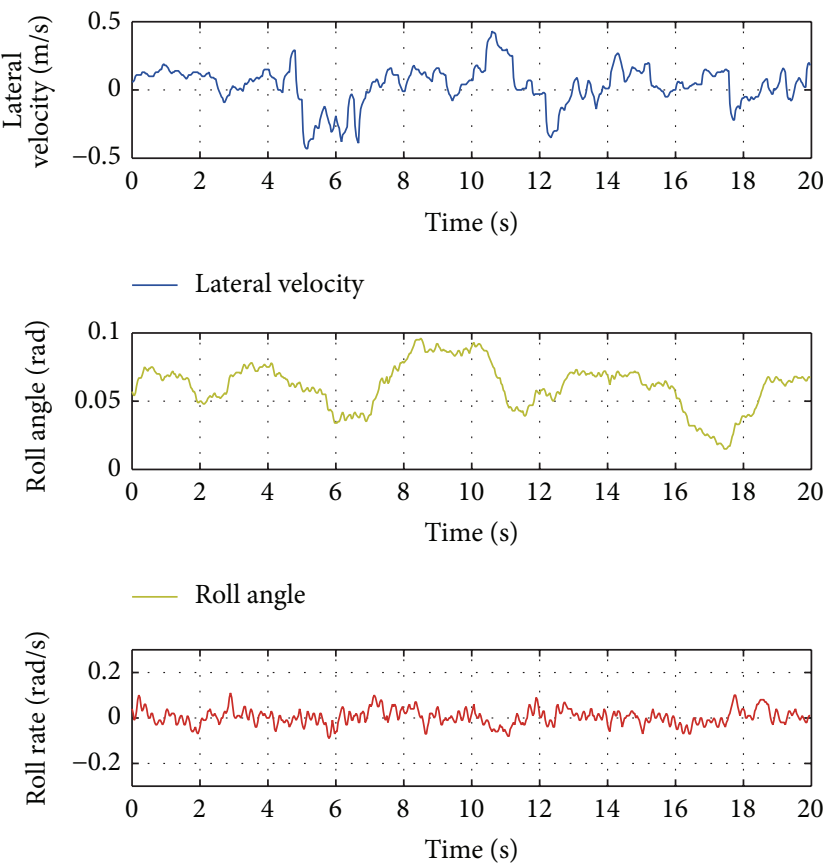

_ Roll rate

FIgURE 9: The lateral output of actual flight.

\section{Conclusion}

This paper first addressed the system identification and the linearized modeling of lateral and longitudinal dynamics of small-scale helicopter. To identify the system of helicopter in a frequency domain method, we used a frequency sweep signal to excite the helicopter around the frequency range of interest. Then, the parameters of the model could be derived 

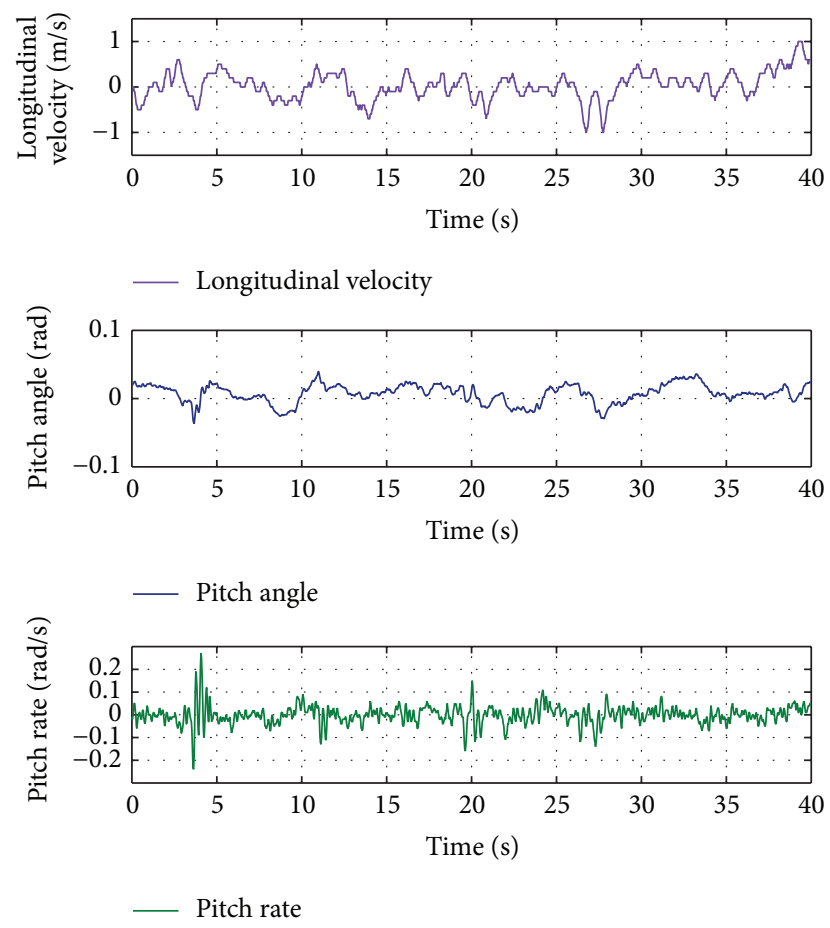

FIGURE 10: The longitudinal output of actual flight.

based on the data from the flight tests, and a verification was conducted to indicate reliability of identification and modeling.

With model of these two channels, $H_{\infty}$ state feedback controller was designed not only to simply stabilize the helicopter in these two directions but also to diminish the extraneous disturbances. The advantage of the state feedback controller is statical and easy to fulfil in actual flight.

Finally, both of the simulation results and the actual flight tests demonstrated a good performance. The other problem that we should discuss next is mainly about how to design a controller for helicopters under the condition of high speed flying.

\section{Conflict of Interests}

The author declares that there is no conflict of interests regarding the publication of this paper.

\section{Acknowledgments}

This work was supported in part by the Scientific Research Foundation of Guangxi Education Department under Grant nos. KY2015ZD143, KY2015ZD140, and KY2015ZD144, Natural Science Foundation of Guangxi under Grant no. 2014GXNSFAA118310, Key laboratory Breeding Base of the Robot and Welding Technology, National Natural Science Foundation of China under Grant no. 61361006, and Key Laboratory Foundation of Guangxi of Unmanned Aerial Vehicle (UAV) Remote Sensing under Grant no. WRJ2015ZR02.

\section{References}

[1] V. Gavrilets, Autonomous aerobatic maneuvering of miniature helicopters [Ph.D. thesis], Massachussetts Institute of Technology, 2003.

[2] B. Mettler, C. Dever, and E. Feron, "Identification modeling, flying qualities and dynamic scaling of miniature rotorcraft," in Proceedings of the NATO System Concepts and Integration (SCI) Symposium, Berlin, Germany, May 2002.

[3] S. Saripalli and G. S. Sukhatme, "Landing on a moving target using an autonomous helicopter," in Proceedings of the International Conference on Field and Service Robotics (FSR '03), Mount Fuji, Japan, 2003.

[4] C. D. Yang and W. H. Liu, "Nonlinear H-innity decoupling hover control of helicopter with parameter uncertainties," in Proceedings of the American Control Conference, pp. 3454-3459, Denver, Colo, USA, June 2003.

[5] X. Wang, Y. Chen, G. Lu, and Y. Zhong, "Robust attitude tracking control of small-scale unmanned helicopter," International Journal of Systems Science, vol. 46, no. 8, pp. 1472-1485, 2015.

[6] S. Jackson, J. Tisdale, M. Kamgarpour, B. Basso, and J. K. Hedrick, "Tracking controllers for small UAVs with wind disturbances: theory and flight results," in Proceedings of the 47th IEEE Conference on Decision and Control (CDC '08), pp. 564-569, Cancun, Mexico, December 2008.

[7] Y. Ma, B. Huang, and C. Xiang, "Forward flight attitude control of unmanned small-scaled gyroplane based on synthesis," in Proceedings of the International Conference on Unmanned Aircraft Systems (ICUAS '15), pp. 1338-1345, IEEE, Denver, Colo, USA, June 2015.

[8] W. Wei and K. Cohen, "Development of a model based fuzzyPID controller for the AeroQuad cyclone quad-copter," AIAA 2015-2029, 2015.

[9] B. Mettler, M. B. Tischler, and T. Kanade, "System identification modeling of a small-scale unmanned rotorcraft for flight control design," Journal of the American Helicopter Society, vol. 47, no. 1, pp. 50-63, 2002.

[10] J. Alvarenga, N. I. Vitzilaios, K. P. Valavanis, and M. J. Rutherford, "Survey of unmanned helicopter model-based navigation and control techniques," Journal of Intelligent \& Robotic Systems, pp. 1-52, 2014.

[11] A. S. Imam and R. Bicker, "Quadrotor model predictive flight control system," International Journal of Current Engineering and Technology, vol. 4, no. 1, pp. 355-365, 2014.

[12] G. Lu, "Aggressive attitude control of unmanned rotor helicopters using a robust controller," Journal of Intelligent \& Robotic Systems, 2014.

[13] M. Bisgaard, A. la Cour-Harbo, and K. A. Danapalasingam, "Nonlinear feedforward control for wind disturbance rejection on autonomous helicopter," in Proceedings of the 23rd IEEE/RSJ 2010 International Conference on Intelligent Robots and Systems(IROS '10), pp. 1078-1083, Taipei, Taiwan, October 2010.

[14] U. B. Hald, M. V. Hesselbak, and M. Siegumfeldt, Nonlinear modeling and optimal control of a miniature autonomous helicopter [M.S. thesis], Aalborg University, Aalborg, Denmark, 2006.

[15] R. A. Hess, "Aircraft and rotorcraft system identificationengineering methods with flight test examples, second edition," Journal of Guidance, Control, and Dynamics, vol. 36, no. 4, pp. 1249-1250, 2013.

[16] D. F. Bassi and W. Fink, "Optimal attitude control parameters via stochastic optimization framework for autonomous 
aircraft," in Proceedings of the IEEE Aerospace Conference, March 2009.

[17] B. Mettler, M. B. Tischler, and T. Kanade, "Attitude control optimization for a small-scale unmanned helicopter," in Proceedings of the AIAA Guidance, Navigation and Control Conference, Denver, Colo, USA, August 2000.

[18] D. H. Shim, H. J. Kim, and S. Sastry, "Control system design for rotorcraft-based unmanned aerial vehicles using time-domain system identification," in Proceedings of the IEEE International Conference on Control Applications, pp. 808-813, IEEE, Anchorage, Alaska, USA, 2000.

[19] C. Ivler and M. B. Tischler, "System identification modeling for flight control design," in Proceedings of the AHS Specialists Meeting on Rotorcraft Handling-Qualities, Liverpool, UK, November 2008.

[20] C. Ivler and M. B. Tischler, "Control system development and flight test experience with the MQ-8B fire scout vertical takeoff unmanned aerial vehicle," in Proceedings of the American Helicopter Society 63rd Annual Forum, Virginia Beach, Va, USA, May 2007.

[21] X. Wang, X. Zhao, and M. Tan, "Modeling, identification and robust control of yaw dynamics of small-scale unmanned helicopters," in Proceedings of the 5th International Conference on Natural Computation (ICNC'09), pp. 273-276, IEEE, Tianjin, China, August 2009.

[22] H.-C. Kim, H. R. Dharmayanda, T. Kang, A. Budiyono, G. Lee, and W. Adiprawita, "Parameter identification and design of a robust attitude controller using $H_{\infty}$ methodology for the raptor E620 small-scale helicopter," International Journal of Control, Automation and Systems, vol. 10, no. 1, pp. 88-101, 2012.

[23] J. Gadewadikar, F. L. Lewis, K. Subbarao, K. Peng, and B. M. Chen, "H-infinity static output-feedback control for rotorcraft," Journal of Intelligent and Robotic Systems: Theory and Applications, vol. 54, no. 4, pp. 629-646, 2009.

[24] K. Zhou, J. Doyle, and K. Glover, Robust and Optimal Control, Prentice Hall, Upper Saddle River, NJ, USA, 1995. 

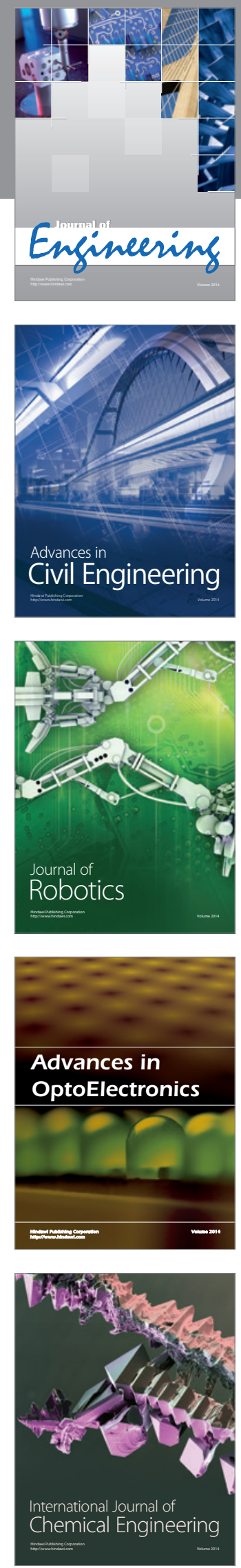

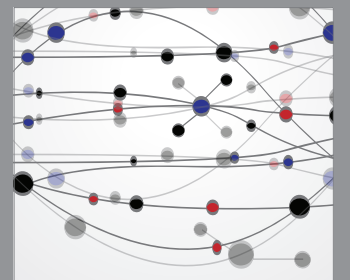

The Scientific World Journal
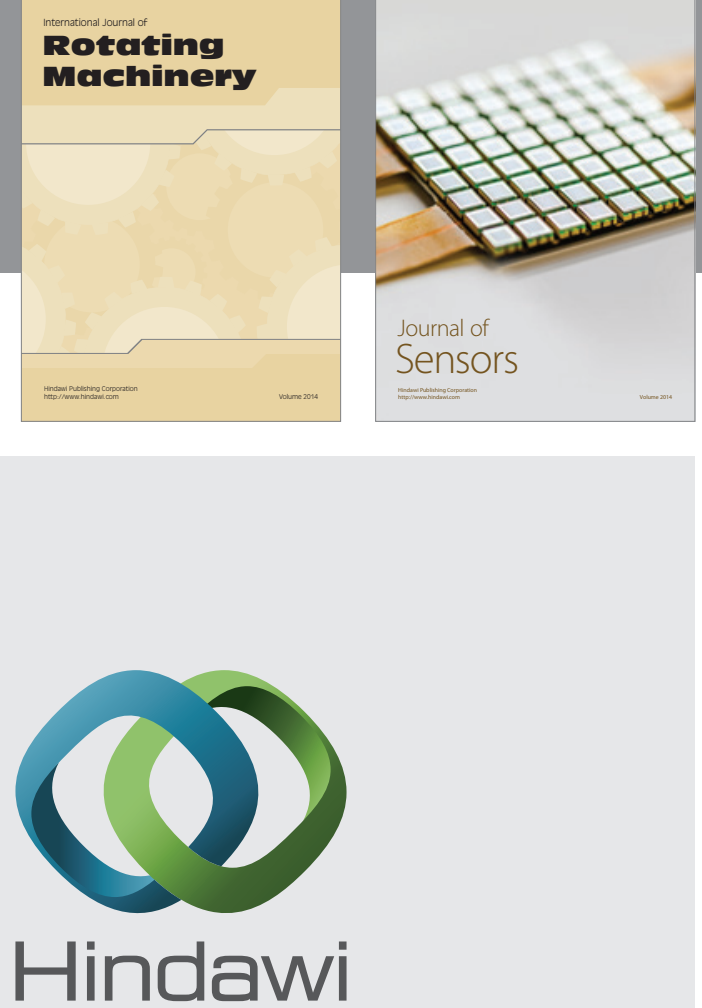

Submit your manuscripts at http://www.hindawi.com
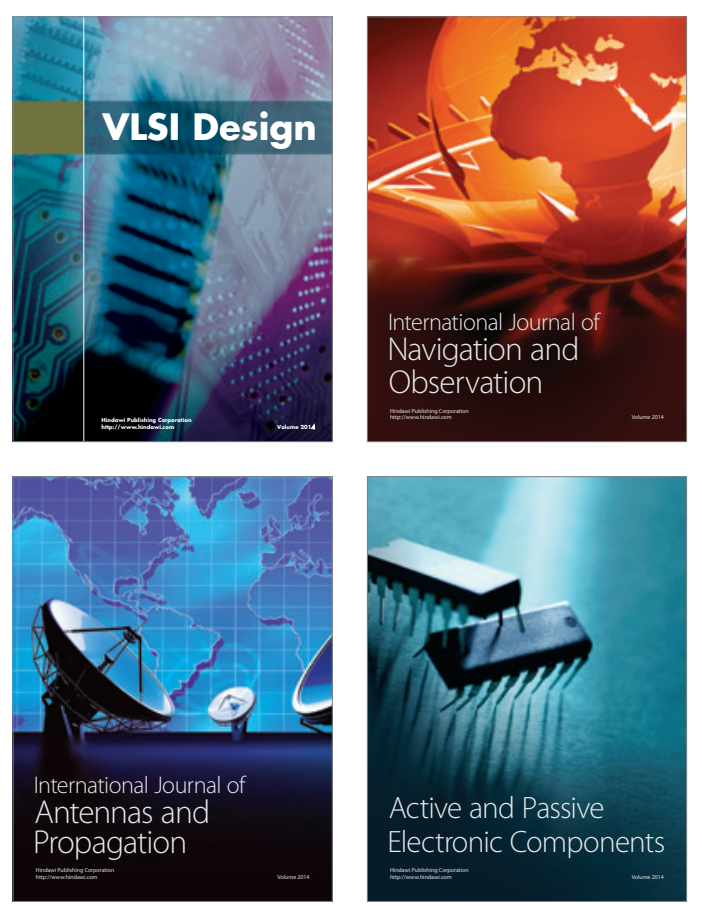
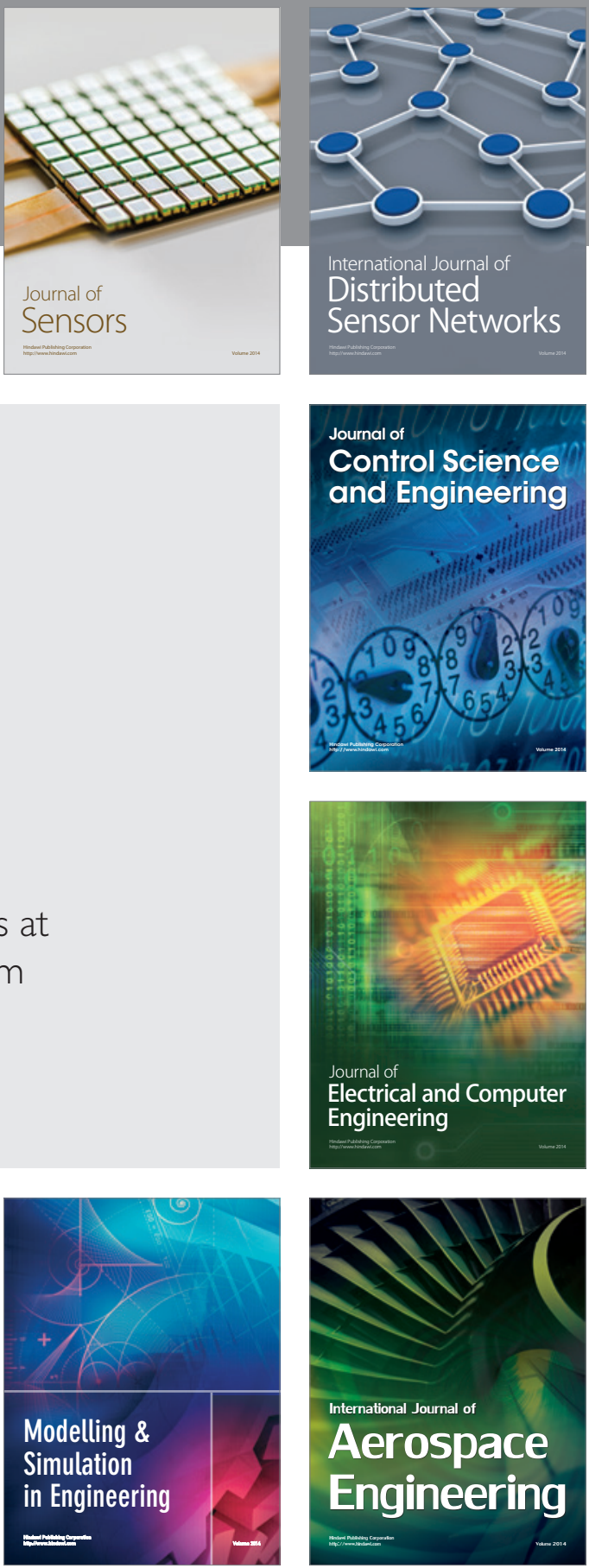

Journal of

Control Science

and Engineering
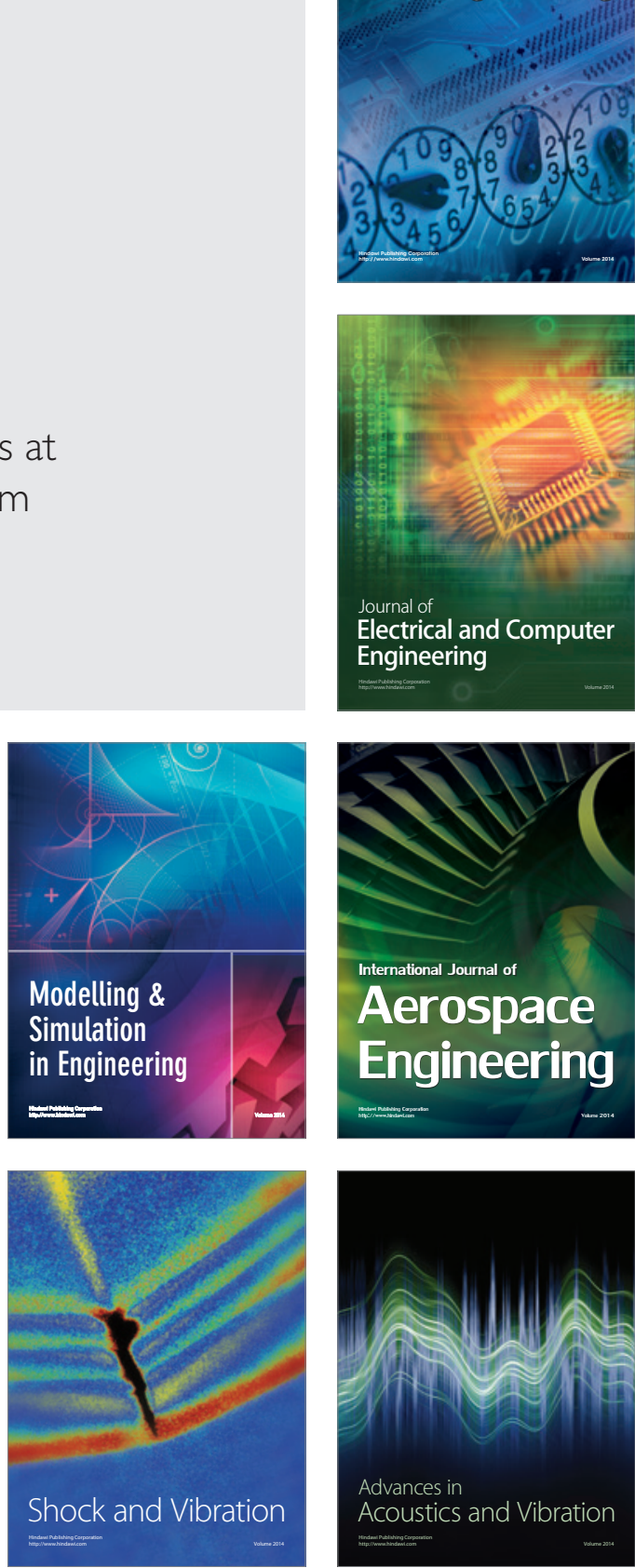